\title{
Politique
}

\section{Sommaire des thèses 1987-1988}

Numéro 14, automne 1988

Sport et politique et Le NPD

URI : https://id.erudit.org/iderudit/040616ar

DOI : https://doi.org/10.7202/040616ar

Aller au sommaire du numéro

Éditeur(s)

Société québécoise de science politique

ISSN

0711-608X (imprimé)

1918-6584 (numérique)

Découvrir la revue

Citer ce document

(1988). Sommaire des thèses 1987-1988. Politique, (14), 211-217.

https://doi.org/10.7202/040616ar

Ce document est protégé par la loi sur le droit d'auteur. L'utilisation des services d'Érudit (y compris la reproduction) est assujettie à sa politique d'utilisation que vous pouvez consulter en ligne.

https://apropos.erudit.org/fr/usagers/politique-dutilisation/
Cet article est diffusé et préservé par Érudit.

Érudit est un consortium interuniversitaire sans but lucratif composé de l’Université de Montréal, l'Université Laval et l'Université du Québec à Montréal. Il a pour mission la promotion et la valorisation de la recherche. https://www.erudit.org/fr/ 


\section{Sommaire des thèses 1987-1988}

\section{Université de Montréal}

Dalpé, Robert, Innovation et politique technologique dans une petite économie industrialisée ouverte n'exerçant pas le leadership technologique, le cas canadien.

L'innovation dans l'industrie peut s'expliquer par l'État de la concurrence et les caractéristiques du marché, mais aussi par les politiques. L'intervention de l'État a la capacité de modifier le fonctionnement de l'entreprise et son environnement. En ce sens, la politique technologique contribue au choix d'une stratégie technologique. Les politiques doivent permettre à l'entreprise de réaliser sa stratégie technologique et l'innovation est alors déterminée par I'orientation des politiques.

La recherche porte sur l'industrie canadienne. Étant donné que la majorité des industries adoptent une stratégie technologique de suiveur, les politiques appliquées seront celles qui permettent les transferts de technologie. Dans les industries poursuivant la recherche jusqu'à l'innovation et exerçant un leadership technologique, les politiques seront plutôt orientées vers les grands programmes de recherche.

La méthodologie adoptée est d'étudier la stratégie technologique de l'industrie pour ensuite déterminer comment les politiques y contribuent. Trois axes décrivent les orientations de la politique technologique.

La politique des communications s'applique aux industries des télécommunications et de l'aérospatiale qui exercent un leadership technologique. Les principaux mécanismes sont la réglementation, la recherche gouvernementale et les politiques d'achat.

Le développement technologique des entreprises suiveuses se fait en grande partie par des transferts. L'économie canadienne est en effet très ouverte et l'industrie importe massivement la technologic étrangère. Les mécanismes de transfert sont notamment l'importation de machinerie, l'échange de service et l'immigration de personnel qualifié. Les multinationales contrôlent la circulation internationale parce qu'elles sont les plus grandes productrices et que beaucoup de transferts sont intra-firmes. La politique des transferts comprend surtout la politique des investissements étrangers qui encadre l'activité des multinationales. La politique des transferts regroupe une série de mécanismes comme les tarifs, les politiques d'achat et les accords de commerce. Ces mécanismes favorisent les transferts de technologie vers l'industrie canadienne.

Le développement des industries des ressources se fait par les programmes de mise en valeur des richesses naturelles. Les sociétés d'État y contribuent en se chargeant des infrastructures de transport et de l'exploitation de l'énergie. 
Larochelle, Gilbert, «L'imaginaire technocratique: La Commission Trilatérale et sa définition d'un nouvel être ensemble."

Cette étude propose un réexamen, voire un renversement des facteurs qui déterminent la pratique sociale de la modernité en regard de son expression technologique contemporaine. Elle vise à montrer que la causalité des conduites et des phénomènes propres à la civilisation occidentale, loin de toujours répondre au constat wébérien d'une rationalisation irréversible, procède parfois d'un mode d'appréhension redevable de l'imaginaire et non de la science. Or, cette occurrence survient dans le cas particulier du discours technocratique dont les agents investissent d'une dimension symbolique la fonctionnalité des moyens matériels pour pouvoir faire accepter de nouveaux cadres sociaux. Et les légitimités qui s'ensuivent proclamées au nom de la science et d'un savoir positif, permettent l'exercice d'un contrôle social. La présente réflexion consiste en ce sens à étudier non seulement le rapport entre la science et l'imaginaire, l'objectivité et la subjectivité, mais aussi entre les évidences sociales et le jeu des intérêts qui les sous-tendent. Son objectif est de démontrer les propositions suivantes:

1- Toute société est fondée sur un imaginaire collectif qui lui sert de référent.

2- Plus une société pense à traves ses référents, moins elle peut penser ses référents en eux-mêmes.

3- La représentation technocratique de la technique constitue un imaginaire collectif dans la société occidentale.

Le parcours de cette démonstration repose sur trois axes fondamentaux: construction d'un schéma théorique autour de la notion d'imaginaire d'une part, mise en relation conceptuelle avec les notions de technique et de technocratie dautre part et, enfin, vérification dans le champ des relations internationales, précisément dans le discours proposé par la Commission Trilatérale. L'observation a conduit à montrer que le projet de cette dernière d'une communauté transnationale - constituée des pays de l'Amérique du Nord, de l'Europe de l'ouest et du Japon - réactualise, même s'il se veut l'expression de la mondialisation des objets techniques, un imaginaire collectif particulier à la civilisation occidentale.

Nadeau, Richard, Modélisation et analyse empirique des fluctuations de court terme de comportement électoral: le cas canadien.

La modélisation des fluctuations de court terme du comportement électoral a donné lieu à la publication d'un nombre impressionnant de travaux depuis une quinzaine d'années. Ces recherches soulèvent des questions importantes. Au premier rang figurent celles de l'incidence de la conjoncture économique sur la décision électorale et du lien entre l'état de l'opinion publique et l'orientation de la politique économique. Ces études débordent toutefois les questions économiques et renvoient à un certain nombre d'interrogations sur les paramètres de la décision électorale, sur l'interaction entre élus et électeurs et, ultimement, sur la portée et la signification du processus électoral.

Une revue des écrits sur la question nous a permis d'apprécier les progrès méthodologiques accomplis. Elle nous a cependant amené à déceler deux lacunes des études 
existantes. La première a trait à la modélisation des déterminants politiques de la popularité. La seconde concerne l'instabilité des relations observées. À cela s'est greffée une troisième constatation: l'absence quasi totale d'études portant sur l'électorat canadien. L'objet de la présente étude a donc consisté à suggérer une version bonifiée de la fonction de la popularité aux fins de l'étude des fluctuations de court terme de l'électorat canadien depuis l'après-guerre.

Les résultats obtenus confirment l'impact des conditions économiques sur la décision électorale. La variable chômage est celle qui a affiché le comportement statistique le plus robuste. Cependant, les relations observées sont faibles. C'est plurôt la prépondérance des variables politiques qui ressort de la présente étude. À ce chapitre, un bref examen des déterminants les plus importants permet de constater que ceux-ci renvoient aux trois objets du soutien politique définis par Easton.

Notre étude voulait d'abord et avant tout mieux cerner les dimensions de la décision électorale. L'examen des fluctuations de la popularité des trois principaux partis politiques fédéraux depuis l'après-guerre, et l'emploi à cette fin du concept de fonction de popularité, débouche sur une meilleure connaissance de la dynamique électorale de court terme au Canada. Cependant, les résultats obtenus et les limites de notre démarche suggèrent que les percées à venir viendront d'une modélisation plus fine de l'interaction entre les variables politiques et économiques et d'une meilleure compréhension du processus de formation de l'agenda politique.

Ouimet, Gérard, Étude de la stratification sexuelle en Europe de l'Est au début des années quatre-vingt.

En dépit du fait que plusieurs études traitent des disparités sociales présentes en URSS, l'attention consentie à l'impact de l'instauration au lendemain de la Seconde Guerre mondiale des régimes communistes en Europe de l'Est sur l'évolution du rôle social des femmes demeure passablement marginale. En fait, la condition féminine s'écarte des préoccupations de recherche spécifiques aux études portant sur l'égalité sociale essentiellement parce que d'une part l'unité d'analyse communément sélectionnée est la famille et, d'autre part, le statut social de la famille est considéré être entièrement déterminé par l'occupation du mari. Quant aux rares études portant sur le statut social des femmes et l'égalité entre les sexes en Europe de l'Est, celles-ci analysent généralement une seule dimension de la vie sociale des femmes et n'établissent qu'occasionnellement une comparaison entre les divers pays de la région.

Il s'avère donc impératif dans un premier remps de s'interroger sur l'existence possible entre les différents pays de l'Europe de l'Est d'une variation concernant leur degré respectif de stratification sexuelle.

Dans un second temps, il importe de circonscrire la nature véritable du changement observé au niveau du statut social des femmes. Ce changement se manifeste-t-il dans toutes les dimensions de la stratification sexuelle? Y a-t-il égalité sexuelle unidimensionnelle ou multidimensionnelle?

Étant donné d'une part que la théorie marxiste analyse les causes des variations spatio-temporelles de la stratification sexuelle et, d'autre part, que la problématique de la présente thèse traite expressément de l'intensité et de la nature d'une telle stratification, il apparaît indiqué, pour les fins heuristiques poursuivies, de s'en remettre à cette théorie. 
De plus, le relevé de littérature scientifique ayant trait aux diverses théories de la stratification sexuelle indique indéniablement que la théorie marxiste s'avère la plus appropriée pour les études réalisées dans le domaine spécifique des sciences politiques.

Finalement, le cadre d'analyse étant les pays de l'Europe de l'Est au début des années quatre-vingt, l'utilisation de la théorie marxiste apparaît totalement justifiée afin de critiquer convenablement l'idéologie officielle de ces pays en matière de condition féminine ainsi que l'application de politiques sociales émanant de cette idéologie commune.

Selon la théorie marxiste, l'instauration de l'égalité sexuelle requiert trois conditions de base, à savoir: la suppression de la propriété privée dont la distribution inéquitable est à l'origine des antagonismes de classes et conséquemment de l'exploitation des travailleurs; l'intégration des femmes sur le marché du travail, leur permettant ainsi d'assumer un rôle social et de jouir d'une indépendance économique; et la socialisation des obligarions familiales (soins des enfants et entretien de la maison) accordant par le fait même aux femmes la possibilité de parfaire davantage leur formation professionnelle et de participer plus activement à la vie publique.

Par ailleurs, l'actualisation des conditions fondamentales de l'émancipation des femmes implique d'une part l'établissement d'un régime socialiste procédant à l'abolition de la propriété privée et, d'autre part, la présence à l'intérieur de ce régime d'un niveau de développement économique suffisamment élevé afin de rendre possible l'entrée massive des femmes sur le marché du travail et la socialisation des obligations familiales.

Tous les pays de l'Europe de l'Est ont adopté un régime socialiste basé sur l'idéologie marxiste-léoniniste. Toutefois, ces mêmes pays different entre eux concernant le niveau de développement économique. En se référant à l'indice statistique - le produit national brut par habitant - établissant quantitativement le niveau d'un tel développement, il est possible de formuler la première hypothèse.

$1^{\circ} \quad$ Le degré de stratification sexuelle en RDA et en Tchécoslovaquie est inférieur à celui observé dans les autres pays de l'Europe de l'Est, à savoir: l'Albanie, la Bulgarie, la Hongrie, la Pologne, la Roumanie, et la Yougoslavie.

Par ailleurs, la théorie marxiste affirme que l'aliénation humaine est un phénomène global, également présent dans toutes les dimensions de la vie des individus dont les conditions matérielles d'existence sont déterminées par les exigences du mode de production. Une telle affirmation signifie que l'égalité sexuelle est un phénomène unidimensionnel, c'est-à-dire que peu importe les sphères d'activités, les rapports entre les sexes sont sensiblement les mêmes. Conséquemment, la deuxième hypothèse est la suivante:

$2^{\circ} \quad$ Le changement dans une dimension de l'égalité sexuelle implique des changements analogues dans d'autres dimensions.

La vérification des hypothèses s'effectuera au moyen de l'analyse secondaire. $\mathrm{Ce}$ type de méthode de recherche, également appelé recréation d'information, consiste à adapter l'information déjà existante afin d'atteindre les objectifs fixés par la problématique retenue. Le matériel de recherche englobe les divers recueils statistiques des pays de l'Europe de l'Est et de l'URSS ainsi que ceux de certains pays et organismes occidentaux. De plus, l'analyse a recours également aux données émanant des recherches en sciences sociales effectuées tant par des chercheurs est-européens et soviétiques que par des chercheurs occidentaux. 
Suite au développement analytique de la présente thèse, il est possible de constater que les deux hypothèses sont en majeure partie infirmées. Il semble que l'explication de la non-confirmation intégrale de ces deux hypothèses, c'est-à-dire l'émergence au sein de chacun des pays de l'Europe de l'Est d'un même pattern inégal de changement entre les différentes dimensions de l'égalité sexuelle, réside dans la présence de variables communes à tous ces pays minant l'avancement de la condition féminine, à savoir: la nature du pouvoir politique, le mode de développement économique, l'acculturation socialiste et l'orientation des politiques sociales. De plus, ces variables, situées dans un contexte d'édification du socialisme, soulignent pertinemment l'inadéquation de la théorie marxiste à l'endroit de l'émancipation des femmes. En fait, la théorie marxiste analysant essentiellement la dynamique «sociétale» en termes de rapport de production, réduit l'appréhension du réel à une seule perspective: celle de la classe ouvrière masculine. La condition féminine apparaît dès lors n'être qu'un problème secondaire, sous-jacent à l'exploitation des travailleurs.

Rocher, François, Rapports de pouvoir et intégration économique continentale 1960-1985: pour une analyse des rapports entre la situation de dépendance, la classe dominante et l'État au Canada.

Notre étude porte sur les intérêts des différentes fractions de la bourgeoisie canadienne et des alliances conclues en vue de favoriser l'intégration économique continentale, du Pacte de l'automobile au débat entourant l'orientation libre-échangiste du début des années 1980.

L'adoption d'une politique commerciale favorisant l'approfondissement des relations économiques continentales reçoit un accueil différencié selon chacune des fractions de la classe dominante. Nos hypothèses de départ étaient à l'effet que les clivages au sein de la bourgeoisie pourraient se présenter de la façon suivante: la fraction compradore serait favorable à une libéralisation des échanges canado-américains alors que les éléments autochtones afficheraient une position beaucoup plus éclatée dans la mesure où les entreprises à caractère monopoliste seraient favorables à une telle option alors que les entreprises non-monopolistes s'y opposeraient ou formuleraient des réserves importantes visant à maintenir les mesures protectionnistes ou des soutiens publics sectoriels. Par ailleurs, les différents groupes représentant les classes subalternes devraient s'opposer à un accroissement de la dépendance économique du Canada à l'égard des États-Unis.

Notre démarche nous a conduit à formuler des indications théoriques sur la nature des rapports qui lient les intérêts de la classe économiquement dominante et l'État. De plus, nous avons spécifié la notion de dépendance, telle qu'elle peut jeter un éclairage sur la situation propre à la formation sociale canadienne, dans le but de tenir compte à la fois des mécanismes économiques et sociaux qui caractérisent une situation concrète de dépendance. La prise en compte des facteurs internes qui rendent compte du processus d'approfondissement du rapport de dépendance s'est réalisée par une étude des positions publiques des principales organisations patronales et syndicales à l'occasion des débats entourant l'adoption du Pacte de l'automobile ainsi que lors des discussions portant sur le projet de libre-échange à compter de la fin des années 1970 et de la première moirié de la décennie 1980 .

Il ressort de cette étude que les clivages au sein de la bourgeoisie ne se font pas en fonction du caractère national ou étranger des entreprises ni selon le schéma monopoliste 
ou non monopoliste. C'est essentiellement la nature des marchés, soit l'orientation vers l'exportation ou non, qui entre en ligne de compte. L'accroissement du rapport de dépendance répond aux intérêts des firmes qui sont déjà intégrées au marché continental et qui cherchent à élargir leur marché, tant intérieur qu'extérieur. Par ailleurs, les porte-parole des classes subalternes s'opposent à l'accroissement des rapports économiques bilatéraux à cause des bouleversements appréhendés sur les structures économique, politique et sociale du Canada.

Salée, Daniel,

Changement social et formation de l'État: Éléments pour une socio-génèse de l'État Canadien (1760-1867)

Les pratiques qui, dès 1867, fixent un certain type d'exercice du pouvoir d'État, une certaine manière de vivre politiquement au Canada, ne s'inscrivent pas en dehors du temps. Elles émanent d'un processus historique qu'il est important de connaître pour mieux saisir leur sens, leur logique propre et les limites de notre conscience collective. L'historiographie a préféré mettre en lumière les idiosyncrasies des Pères de la Confédération ou les calculs économiques d'une certaine bourgeoisie pour expliquer la formation de l'État canadien. On ne sait rien ou presque sur la philosophie sociale qui informe au dixneuvième siècle la réalitéétatique canadienne; sur la rationalité politique et socio-culturelle sous-jacente à la mise sur pied de l'État canadien moderne. La thèse propose une réflexion sur ce sujet.

La perspective d'analyse qui y est adoptée se démarque sensiblement des interprétations qui associent la création de l'État canadien à la montée du capitalisme comme mode dominant d'organisation socio-économique. Elle repose sur la conviction que la structure étatique nouvelle ne s'impose pas comme simple reflet du capitalisme naissant, mais comme solution à une crise qui, fondamentalement, est de nature socio-culturelle.

L'optique privilégiée s'éloigne des analyses de l'État faites à partir d'un examen de sa matérialité institutionnelle. L'effort analytique cherche plutôt à mettre en relief la manière dont s'incarne la problématique du pouvoir et de la domination au sein de la société. L'intelligence de l'historicité de l'État ne peut être mise à jour que par la compréhension des rapports sociaux tels qu'ils sont enferrés dans la logique de la domination. Les rapports sociaux fixent des pratiques sociétales qui, de par leur antagonisme, rendent le mouvement historique: le sens historique de l'État émerge de lui-même du sens de ces pratiques.

La thèse articulera trois niveaux de réflexion interreliés:

1. Formuler un cadre théorique propre à l'analyse du changement socio-politique à l'intérieur de saciétés dont le passé colonial est similaire à celui du Canada. Il émane de l'imagination sociologique une compréhension de l'historicité des sociétés comme le Canada fondée presque entièrement sur l'action économique marchande de formations sociales métropolitaines qui les marquent conjoncturellement. Une telle attitude conduit l'analyse socio-historique des colonies (ou ex-colonies) à reléguer au second plan, voire à délaisser complètement toute prise en considération des processus sociétaux auto-centrés. La situation de dépendance économique ne doit pas obscurcir le fait que les formations sociales coloniales s'animent d'une dynamique sociale interne, d'un «entrechoc" de forces sociales, qui leur est propre et au sein duquel apparaissent en creux les modalités de l'historicité de la société et de l'État. 
2. Améliorer notre connaissance générale de l'historicité du capitalisme au Canada pour déboucher sur une conceptualisation renouvelée du mouvement bistorique. L'analyse et l'étude du capitalisme canadien participe d'une saisie téléologiste et linéaire de son mouvement. L'inexorabilité qui, conceptuellement, lui est conférée justifie qu'on le considère comme seul substrat d'actualisation de la société, le seul dont l'analyse ait à rendre compte. À tort cependant, puisque le capitalisme est une réponse conjoncturelle à une réalité sociétale historiquement déterminée: il ne se produit pas par génération spontanée et il faut pouvoir le conceptualiser dans la foulée d'un ordre socio-économique qui lui est antérieur. Ce passage d'un ordre socio-économique à un autre n'a rien de pré-établi: il s'accomplit selon un processus complexe, inscrit dans la configuration des rapports sociaux. Lanalyse doit donc rester attentive aux situations concrètes où le capitalisme n'agit pas comme mode dominant d'opération et de développement d'une société et concevoir l'articulation possible du capitalisme avec quelque socio-économie non capitaliste.

3. Interroger le caractère socio-bistorique de l'exercice du posvoir d'État au Canada afin d'éclairer les modulations contemporaines de la culture politique canadienne. Comment expliquer qu'en dépir de son enveloppe fédérative, la structure étatique canadienne ait imposé très rapidement une nette tendance au centralisme politico-administratif et à l'interventionnisme socioéconomique? Des travaux ont bien cherché dans l'idée d'un fragment idéologique défini selon les idiosyncrasies des premiers arrivants quelque réponse; mais il ne suffit pas d'invoquer l'inclination historiquement tory de la culture politique canadienne pour rendre compte du caractère interventionniste de l'État fédéral ou du respect des citoyens pour les autorités gouvernementales. Il faut faire enquête sur l'évolution des processus sociétaux qui sous-tendent l'actualisation de l'État et de son pouvoir afférent. Il s'agit d'explorer l'historicité sociétale de l'État canadien; faire l'archéologie socio-politique de notre dixneuvième siècle.

\section{Université McGill}

Rejali, Darius M. Discipline and Torture, Or How Iranians Because Moderns.

In this dissertation, I undertake an empirical analysis of Iranian punitive practices over the last century. In this context, I set out to investigate three issues. First, I critically examine the claim that modernity is characterized by a diminution of corporal punishments, considering both the older humanist-progressivist version of this claim and the revisionistNietzschean versions that have been advanced by several scholars including Michel Foucault, David Rothman, Michael Ignatieff, John Langbein, Gerhard Oestreich, and Marc Raeff. In particular, I examine the relationship between modern torture and what might be called the "disciplinary process" that is said to characterize modernization. Second, I evaluate Chomsky and Herman's hypothesis that developing societies are characterized by a specific economy of violence that might be described as «state terrorism. " Third, I test the urility of Foucault's theoretical approach to the study of power. 